\title{
To Study the Outcome of Posterior Sagittal Anorectoplasty in Anorectal Malformations
}

\author{
SHAFIQ-UR-REHMAN ${ }^{1}$, MUHAMMAD ANWAR $^{2}$ \\ ${ }^{1}$ Assistant Professor of Paediatric Surgery, Sahiwal Medical College Sahiwal \\ ${ }^{2}$ Associate Professor of Paediatrics, Rashid Latif Medical College, Lahore \\ Correspondence to Dr. Shafiq-ur-Rehman E-mail: shafiqlangriyal@gmail.com, Cell: 0300-9695031
}

\begin{abstract}
Aim: To assess the effectiveness of posterior sagittal anorectplasty for the repair of anorectal malformations in terms of postoperative complications and functional outcomes.

Study design: Descriptive prospective study.

Place and duration of study: Sahiwal Teaching Hospital, Sahiwal. From $1^{\text {st }}$ January 2016 to $31^{\text {st }}$ December 2019.

Methodology: Forty-two children with high/intermediate anorectal malformations, who underwent posterior sagittal anorectoplasty, were included in the study. Patients with low variety anorectal malformations, recto vestibular/ano vestibular fistula, persistent cloaca and patients with sacral dysraphism, spinal dysraphism, myelomeningocele spina bifida occulta and menifesta were excluded. Demographic information included age, gender, weight, type of anorectal malformation, type of surgery performed, operation time and hospital stay. Immediate post-operative complications were also noted. Bowel habits, constipation and faecal incontinence were evaluated in all patients during the follow up period.

Results: Thirty-one (73.80\%) were males and $11(26.19 \%)$ were females and mean age was 1.72 years. The mean weight at the time of operation was $11.00 \mathrm{~kg}$. High variety anorectal malformations were $14(33.33 \%)$ and intermediate were $28(66.66 \%)$. Rectourethral fistula 25(59.52\%) was most common variety seen in males. Recto bulber urethral fistula in $17(40.47 \%)$ and recto prostatic urethral fistula in $8(19.04 \%)$ patients were seen. Anal agenesis without fistula $7(16.66 \%)$ was most common anomaly in females. Overall complication rate was $15(35.71 \%)$. Constipation $11(26.19 \%)$ was most common post-operative complication. True fecal incontinence was seen in 3(7.14\%) patients. Strong and effective squeeze of anal sphincter was seen in $39(92.85 \%)$ patients and satisfactory bowel habits were present in $37(88.08 \%)$ patients.

Conclusion: Posterior sagittal anorectplasty is a useful procedure in the management of high/intermediate anorectal malformations, precise identification and reconstruction of muscle complex result in good anatomical and functional outcome. Immediate post-operative complication rate is also low.

Keywords: Anorectal malformations, Posterior Sagittal Anorectoplasty, Postoperative complications, Functional outcomes
\end{abstract}

\section{INTRODUCTION}

Anorectal Malformation (ARM) is one of the most common congenital intestinal anomalies with an incidence of 1 in 4000 to 5000 births and a slightly higher prevalence in boys ${ }^{1}$. It has a wide spectrum of pathoanatomic abnormalities, from minor defects to complex malformation such as persistent cloaca and from isolated anomaly to associations with urogenital, cardiac, oesophageal atresia, gastrointestinal, vertebral, and limb anomalies ${ }^{2,3}$.

Anorectal malformations have been treated since antiquity. The first description of anorectal malformation (ARM) repair dates back to the second century $A D$ by Sorano. Although it was unsuccessful, the first surgical attempt was made in 1783 by performing an inguinal colostomy. Various methods of surgica repair have evolved from first anoplasty by Amussat in 1835, to an abdominal perineal pull through in which bowel was pulled down close to the sacrum and through the puborectalis sling. ${ }^{4}$ Then the first time posterior sagittal approach was used in 1980 and it allowed the direct exposure of this complex anatomic area. ${ }^{5}$ Posterior sagittal anorectoplasty, which was introduced by Pena and de Vries in 1980, is now widely accepted as a standard surgical procedure for the management of anorectal malformations and many published studies have shown better functional outcome and low complication rate after posterior sagittal anorectoplasty, $6,7,8$. Despite its widespread use, poor functional outcomes are still reported after posterior sagittal anorectoplasty ${ }^{9}$.

Traditionally, posterior sagittal anorectoplasty is performed as multistage procedure - temporary diversion colostomy in neonatal period, posterior sagittal anorectoplasty followed by colostomy closure. With the advancement in neonatology, anaesthesiology, and surgical expertise, pediatric surgeons are increasingly focusing on primary posterior sagittal anorectoplasty in neonates. Primary posterior sagittal anorectoplasty performed at birth, without preceding colostomy is safe and feasible ${ }^{10}$.

Received on 25-03-2021

Accepted on 18-08-2021
Laparoscopically assisted anorectal pull through was first reported by Georgeson et al in $2000^{11}$. Due to the minimal invasive nature of laparoscopically assisted anorectoplasty, it provides better cosmetic results, rapid recovery, less pain and shorter hospital stay ${ }^{12}$. Several concerns remain, whether it has better functional outcomes compared with posterior sagittal anorectoplasty ${ }^{13-15}$.

With the evolution of surgical techniques, outcome of patients with anorectal malformations has improved. However, decision regarding the choice of approach and procedure depends on the settings, infrastructure and surgical expertise. In our settings, we do traditional posterior sagittal anorectoplasty in patients with high and intermediate variety of anorectal malformations. This study was carried out to evaluate the procedure in terms of postoperative complications and functional outcomes.

\section{MATERIALS AND METHODS}

This prospective descriptive study conducted in the Department of Paediatric Surgery, Sahiwal Teaching Hospital, Sahiwal, from $1^{\text {st }}$ January 2016 to $31^{\text {st }}$ December 2019 after permission from Ethical Committee. A total of 42 children with high/intermediate anorectal malformations were included. Age ranged from nine months to five years. All patients were managed previously with temporary diversion colostomy in neonatal period. Patients with low variety anorectal malformations, female patients with recto-vestibular/ anovestibular fistula., patients presented with persistent cloaca, sacral dysraphism, spinal dysraphism, myelomeningocele and spina bifida occulta and menifesta were excluded. Pre-operative assessment was done with history, clinical examination and lab investigations. A thorough examination was particularly performed to evaluate the perineal anatomy and sacral defects. Distal loopogram was performed in all patients to evaluate the anatomy of distal loop. Abdominal sonography and plain X-ray spine were advised to assess associated anomalies. Echocardiography was performed only in selected cases. Distal loop preparation was carried out with normal saline. Antibiotics, ceftriaxone and 
metronidazole were started 24 hours before surgery and continued postoperatively for five days. Posterior sagittal anorectoplasty was performed according to the basic principles of posterior sagittal approach, as described by de Vries and Pena. Anal dilatation started two weeks after repair. Divided descending colostomy was closed once desired anal size was reached, usually six to eight weeks after posterior sagittal anorectoplasty. Variables studied included age, gender, weight, type of anorectal malformation, type of surgery performed, operation time and hospital stay. Patients were classified according to international classification of anorectal malformations, into types (high and intermediate) and subtypes Immediate post-operative complications were also noted. Bowe habits (feeling of urge, capacity to report urge, ability to hold back), constipation and faecal incontinence were evaluated according to Krickenbeck classification. Bowel habits were assessed in children above three years of age. Sphincter was assessed according to Kelly's clinical score. The patients were followed in outdoor patient department till they became toilet trained. Minimum follow up period was one year.

All patients were operated under general anaesthesia with endotracheal intubation. Urinary catheter was inserted preoperatively. Patient was placed in prone position with elevated pelvis. An electrical stimulator was used to elicit muscle contraction and site of new anus was identified. Midsagittal skin incision, leaving equal amount of muscle on both sides, was given. The incision exposed the subcutaneous tissue, parasagittal fibers, muscle complex and levator muscle. The sphincter mechanism/muscle complex was divided. The deep pelvic fascia was opened and rectal pouch was identified. Two silk sutures were placed in the posterior rectal wall and rectal pouch was opened longitudinally between the sutures. Once the fistula was identified, separation of rectal pouch was performed by sharp dissection. Initially followed submucosal plan, then the dissection became full rectal wall thickness. Rectum was separated completely from urethra, staying close to the rectal wall. Rectourethral fistula was closed in two layers. Tapering of rectum was performed whenever it was needed. Then rectum was pulled down within muscle complex and standard anoplasty was performed. Wound closed in layers. Statistical analysis was performed using SPSS version 24 Student $t$ test was applied and $p<0.05$ considered as significant

\section{RESULTS}

There were $31(73.80 \%)$ males and $11(26.19 \%)$ females and age ranged from $0.75-5$ years with mean age was 1.72 years. The mean weight at the time of operation was $11.00 \mathrm{~kg}$ (range 8.5 $18 \mathrm{~kg}$ ). Duration of surgery ranged from $80-110$ min (mean $88.50 \mathrm{~min}$ ). Average hospital stay was 5.64 days (range 4-9 days). High variety anorectal malformations were 14 (33.33\%) and intermediate were $28(66.66 \%)$. Rectourethral fistula $25(59.52 \%)$ was most common variety seen in males. Recto bulbar urethral fistula in $17(40.47 \%)$ and recto prostatic urethral fistula in $8(19.04 \%)$ were recorded (Table 1$)$.

Table 1: Types and sub types of anorectal malformations

\begin{tabular}{|c|c|c|}
\hline Gender & High variety & Intermediate variety \\
\hline Male & $10(23.80 \%)$ & $21(50.00 \%)$ \\
\hline Female & $4(9.52 \%)$ & $7(16.66 \%)$ \\
\hline \multicolumn{3}{|c|}{ Types of male anorectal malformations } \\
\hline Type & Sub-type & No. (\%) \\
\hline \multirow[b]{2}{*}{ High variety } & Recto vesical fistula & $2(4.76 \%)$ \\
\hline & $\begin{array}{l}\text { Recto prostatic } \\
\text { urethral fistula }\end{array}$ & $8(19.04 \%)$ \\
\hline \multirow{2}{*}{ Intermediate Variety } & $\begin{array}{l}\text { Recto bulbar } \\
\text { urethral fistula }\end{array}$ & $17(40.47 \%)$ \\
\hline & $\begin{array}{l}\text { Anal agenesis } \\
\text { without fistula }\end{array}$ & $4(9.52 \%)$ \\
\hline \multicolumn{3}{|c|}{ Types of female anorectal malformations } \\
\hline \multirow[b]{2}{*}{ High variety } & Rectal Atresia & $1(2.38 \%)$ \\
\hline & $\begin{array}{l}\text { Anorectal agenesis } \\
\text { without fistula }\end{array}$ & $3(7.14 \%)$ \\
\hline Intermediate Variety & $\begin{array}{l}\text { Anal agenesis } \\
\text { without fistula }\end{array}$ & $7(16.66 \%)$ \\
\hline
\end{tabular}

reported in $11(26.19 \%)$ patients and was most common post- operative complication. Soiling was seen in $2(4.76 \%)$ patients of constipation with fecal impaction. True fecal incontinence was seen in $3(7.14 \%)$ and mucosal prolapse in $4(9.52 \%)$ patients (Table 2). Strong and effective squeeze of anal sphincter was seen in $39(92.85 \%)$ patients. Voluntary bowel habits were assessed in 35 children who were above three years of age and satisfactory bowel habits were present in $30(85.71 \%$ ) patients (Table 3 ).

Table 2: Post-operative complications

Table 2: Post-operative complications
\begin{tabular}{|l|c|c|}
\hline Complication & No. & $\%$ \\
\hline Wound infection & 3 & 7.14 \\
\hline Anal stenosis & 7 & 16.66 \\
\hline Mucosal prolapse & 4 & 9.52 \\
\hline Constipation & 11 & 26.19 \\
\hline Fecal incontinence & 3 & 7.14 \\
\hline
\end{tabular}

Table 3: Assessment of constipation, incontinence and sphincter

\begin{tabular}{|l|c|c|}
\hline Variable & No. & $\%$ \\
\hline Constipation & 2 & 4.76 \\
\hline Grade 1 (manageable by change in diet) & 8 & 19.04 \\
\hline Grade 2 (requires laxatives) & 1 & 2.38 \\
\hline Grade 3 (resistant to laxatives \& diet) & 1 & 2.38 \\
\hline Incontinence & 1 & 2.38 \\
\hline Grade 1 (occasionally, once or twice a week) & 1 & $(2.38$ \\
\hline Grade 2 (every day, no social problem) & 39 & 92.85 \\
\hline Grade 3 (constant, social problem) & 2 & 4.76 \\
\hline Sphincteric function & 1 & 2.38 \\
\hline Strong and effective squeeze & \multicolumn{1}{|l}{} \\
\hline Week and partial squeeze & \multicolumn{1}{|l|}{} \\
\hline No contraction & 1 & \\
\hline
\end{tabular}

\section{DISCUSSION}

Anorectal malformations are very common and complex congenital anomalies with lot of anatomical variations. The management of anorectal malformation has been a cause of major concern to pediatric surgeons all around the world. Despite the advances in surgical techniques, children with anorectal malformations have to face the challenges of bowel, bladder, and sexual dysfunction and psychosocial problems. Numerous operations have been devised for treatment of anorectal malformations. The ultimate aim of surgery is to reconstruct the functional and anatomically aesthetic neo-anal canal and anorectum. Posterior sagittal anorectoplasty enables the paediatric surgeons to assess the distal rectal pouch along with any associated fistulas. It also involves the precise identification and reconstruction of muscle complex around neoanus. This study documents our experience of this technique.

Slight male preponderance is reported in majority of the published literature ${ }^{16-18}$. However, in this study a significant male preponderance was seen. Exclusion of recto vestibular fistula and persistent cloacal from this study may the reason. However similar findings have been reported in some studies conducted in Pakistan. ${ }^{19,20}$ Some social factor, like gender preference, may be the reason. Female anorectal malformations may be under reported. In our study high variety anorectal malformations were $14(33.33 \%)$ and intermediate were $28(66.66 \%)$. In another study high anorectal malformations accounted for 33 (34.4\%), intermediate $15(15.6 \%)$ and low types were $48(50 \%)^{18}$. Rectourethral fistula, $25(59.52 \%)$ was the most common variety seen in males. This finding of our study is consistent with published literature ${ }^{18,19,21}$. Overall complication rate was $15(35.71 \%)$ in the present study. Similar complication rate, $11(33.33 \%)$ reported in one study. ${ }^{18}$ Most of the complications were minor and settled with conservative measures. Wound infection was present in $3(7.14 \%)$ patients. High overall wound infection, $28(31 \%)$ was reported in one study. Nineteen patients $(21 \%)$ had superficial and $9(10 \%)$ deep wound dehiscence. ${ }^{22}$ Patients of single stage primary posterior sagittal anorectoplasty were also included in this study. Low wound infection rate in our study may be due to the presence of colostomy in all patients. Seven patients (16.66\%) developed anal stenosis which was managed conservatively with periodic dilatation. Osagie ${ }^{18}$ reported 
anal stenosis in $3(9 \%)$ patients. All patients responded to closely supervised anal dilatation.

The functional outcomes are subjective symptoms and difficult to interpret. The evolution of different classifications and scoring systems has further increased the difficulty in comparing the functional results. On the other hand, functional outcomes do not depend only on the surgical technique. Perianal innervation, rectal motility and development of muscle complex and pelvic floor muscles also play vital role. Studies of anorectal function after posterior sagittal anorectoplasty have shown varying results. Constipation was the most common functional disorder observed in our study. Eleven patients $(26.19 \%)$ developed constipation, $2(4.76 \%)$ grade 1 and managed with diet modification and toilet training, 8(19.04\%) grade 2 and were treated with laxatives and $1(2.38 \%)$ grade 3 constipation required rectal irrigation and fecal evacuation. Other studies also reported constipation as the most common complication in patients who underwent posterior sagittal anorectoplasty ${ }^{21,23,24}$. Fecal incontinence is most significant longterm problem after anorectal surgery and occurred in $3(7.14 \%)$ patients in this study, two patients with rectovesical fistula and one with recto prostatic urethral fistula. None of the patients had urinary incontinence.

In this study, 30(85.71\%) patients were able to achieve voluntary bowel habits. Pena and Hong ${ }^{25}$ reported that $75 \%$ of patients achieved voluntary bowel movements ${ }^{8}$. De Varies reported that $46(73 \%)$ patients showed good anorectal function. Results from a study by Sukarnjanaprai ${ }^{24}$ also showed that $37(68.3 \%)$ had good voluntary bowel habits. The results of our study are comparable with the recent studies.

\section{CONCLUSION}

Posterior sagittal anorectoplasty is an appropriate procedure in the management of high/intermediate anorectal malformations. Precise identification and reconstruction of muscle complex results in good anatomical and functional outcome. Immediate postoperative complication rate is also low. In the skilled hands it is safe and effective procedure.

\section{Conflict of interest: Nil}

\section{REFERENCES}

1. Cairo SB, Gasior A, Rollins MD, Rothstein DH. Challenges in transition of care for patients with anorectal malformations: a systematic review and recommendations for comprehensive care. Dis Colon Rectum 2018;61(3):390-9.

2. Gupta R, Gupta A, Shukla A, Chaturvedi V, Sharma P, Tanger R. Anorectal malformations: Early outcome analysis from a high-volume tertiary care institute. Medical Journal of Dr DY Patil Vidyapeeth 2019;12(2):122-30.

3. Nisar MU, Khan NA, Javed N, Sikander S, Chaudhry MA, Chaudhry AR. Esophageal Atresia: Management and Outcome in Resource Limited Settings. Pak J Med Res 2019;58(4):159-64.

4. Stephens FD. Imperforate rectum: a new surgical technique. Med J Aust 1953; 1:202-6.

5. Peña A, Devries PA. Posterior sagittal anorectoplasty: important technical considerations and new applications. J Pediatr Surg 26.
6. Levitt MA, Peña A. Anorectal malformations. Fundamentals Pediatr Surg 2011:499-512.

7. Liu G, Yuan J, Geng J, Wang C, Li T. The treatment of high and intermediate anorectal malformations: one stage or three procedures? J Pediatr Surg 2004;39(10):1466-71.

8. Peña A, Hong A. Advances in the management of anorectal malformations. Am J Surg 2000;180(5):370-6.

9. Gangopadhyay AN, Pandey V. Anorectal malformations. J Indian Assoc Pediatr Surg 2015;20(1):10.

10. Tiwari C, Shenoy NS, Gandhi S, Makan A, Basu S, Tulsian A, Shah H. Primary posterior sagittal anorectoplasty in male neonates with anorectal malformations: a tertiary care hospital experience. J Neonat Surg 2021;10:29-33.

11. Georgeson KE, Inge TH, Albanese CT. Laparoscopically assisted anorectal pull-through for high imperforateanus, a new technique. J Pediatr Surg 2000; 35(6): 927-31.

12. Gołębiewski $A$, Murawski $M$, Łosin $M$, Królak $M$, Czauderna $P$. Laparoscopic surgical technique to facilitate management of high anorectal malformations-report of seven cases. Videosurgery and other miniinvasive techniques $2011 ; 6(3): 150$.

13. Ruggeri G, Destro F, Randi B, Lima M. Laparoscopic-assisted anorectal pull-through for high imperforate anus: 14 years experience in a single center. J Laparoendoscopic Advanced Surg Tech 2016;26(5):404-8.

14. Han Y, Xia Z, Guo S, Yu X, Li Z. Laparoscopically assisted anorectal pull-through versus posterior sagittal anorectoplasty for high and intermediate anorectal malformations: a systematic review and metaanalysis. PLoS One 2017;12(1): e0170421.

15. Tainaka T, Uchida H, Tanaka Y, Hinoki A, Shirota C, Sumida W, et al. Long-term outcomes and complications after laparoscopic-assisted anorectoplasty vs posterior sagittal anorectoplasty for high-and intermediate-type anorectal malformation. Pediatr Surg Int 2018; 34(10): 1111-5.

16. Nisar MU, lqbal A, Javed N, Sikander S, Burki SA, Chaudhry MA. Factors affecting the outcome of neonates with anorectal malformation in a developing country. J Pediatr Adolescent Surg 2020;1(2).

17. Tofft L, Salö M, Arnbjörnsson E, Stenström P. Wound dehiscence after posterior sagittal anorectoplasty in children with anorectal malformations. BioMed Res Int 2018; 11: 2018.

18. Osagie TO, Aisien E, Osifo OD. Outcomes of posterior sagittal anorectoplasty for high anorectal malformation in benin city, Nigeria. $J$ West Afr Coll Surg 2016;6(1):16.

19. Qazi SH, Faruque AV, Khan MA, Saleem U. Functional outcome of anorectal malformations and associated anomalies in era of Krickenbeck classification. JCPSP 2016;26(3):204.

20. Mirza B, ljaz L, Saleem M, Sharif M, Sheikh A. Anorectal malformations in neonates. Afr J Paediatr Surg 2011; 8(2):151.

21. Sukarnjanaprai N, Ngerncham M, Laohapensang M. The Functional outcomes in anorectal malformations after posterior sagittal anorectoplasty. J Med Assoc Thai 2017;100(4): S105-13.

22. Tofft L, Salö M, Arnbjörnsson E, Stenström P. Wound dehiscence after posterior sagittal anorectoplasty in children with anorectal malformations. BioMed Res Int 2018; 11;2018.

23. Askarpour S, Ostadian N, Javaherizadeh H, Mousavi SM. Outcome of patients with anorectal malformations after posterior sagittal anorectoplasty: a study from Ahvaz, Iran. Ann Pediatr Surg 2014;10(3):65-7.

24. Huang CF, Lee HC, Yeung CY, Chan WT, Jiang CB, Sheu JC, Wang $\mathrm{NL}$, Lin JR. Constipation is a major complication after posterior sagittal anorectoplasty for anorectal malformations in children. Pediatr Neonatol 2012;53(4):252-6.

25. de Vries PA: Results of treatment and their assessment. In: Stephenes FD, Smith ED, eds. Anorectal malformations in children: Up-date 1988. New York: Alan R. Liss, 1988; 481-500. 